\title{
Using amide proton transfer-weighted MRI to non-invasively differentiate mismatch repair deficient and proficient tumors in endometrioid endometrial adenocarcinoma
}

\author{
Yuan $\mathrm{Li}^{1 \dagger}$, Xinyu Liu ${ }^{2 \dagger}$, Xiaoqi Wang ${ }^{3 \dagger}$, Chengyu Lin², Yafei Qi ${ }^{2}$, Bo Chen ${ }^{4}$, Hailong Zhou², Qiaoling Wu², \\ Jing Ren ${ }^{2}$, Jia Zhao ${ }^{2}$, Junjun Yang ${ }^{1}$, Yang Xiang ${ }^{1}$, Yonglan He $\mathrm{2}^{2^{*}} \mathbb{D}$, Zhengyu Jin ${ }^{2^{*}}$ and Huadan Xue $\mathrm{2}^{2^{*}}$
}

\begin{abstract}
Objectives: To investigate the utility of three-dimensional (3D) amide proton transfer-weighted (APTw) imaging to differentiate mismatch repair deficient (dMMR) and mismatch repair proficient (pMMR) tumors in endometrioid endometrial adenocarcinoma (EEA).

Methods: Forty-nine patients with EEA underwent T1-weighted imaging, T2-weighted imaging, 3D APTw imaging, and diffusion-weighted imaging at 3 T MRI. Image quality and measurement confidence of APTW images were evaluated on a 5-point Likert scale. APTw and apparent diffusion coefficient (ADC) values were calculated and compared between the dMMR and pMMR groups and among the three EEA histologic grades based on the Federation of Gynecology and Obstetrics (FIGO) grading system criteria. Student's t-test, analysis of variance with Scheffe post hoc test, and receiver operating characteristic analysis were performed. Statistical significance was set at $p<0.05$.

Results: Thirty-five EEA patients ( 9 with dMMR tumors and 26 with pMMR tumors) with good image quality were enrolled in quantitative analysis. APTw values were significantly higher in the dMMR group than in the pMMR group (3.2 $\pm 0.3 \%$ and $2.8 \pm 0.5 \%$, respectively; $p=0.019)$. ADC values of the $\mathrm{dMMR}$ and $\mathrm{PMMR}$ groups were $0.874 \pm 0.104 \times 10^{-3} \mathrm{~mm}^{2} / \mathrm{s}$ and $0.903 \pm 0.100 \times 10^{-3} \mathrm{~mm}^{2} / \mathrm{s}$, respectively. No significant between-group difference was noted $(p=0.476)$. No statistically significant differences were observed in APTw values or ADC values among the three histologic grades ( $p=0.766$ and $p=0.295$, respectively).
\end{abstract}

Conclusions: APTw values may be used as potential imaging markers to differentiate dMMR from pMMR tumors in EEA.

Keywords: Amide proton transfer-weighted, Magnetic resonance imaging, Endometrioid endometrial adenocarcinoma, Mismatch repair deficient

*Correspondence: heyonglan@pumch.cn; jin_zhengyu@163.com; bjdanna95@hotmail.com

'Yuan Li, Xinyu Liu and Xiaogi Wang contributed equally to this work 2 Department of Radiology, Peking Union Medical College Hospital, Peking Union Medical College and Chinese Academy of Medical Sciences, Shuai Fu Yuan 1\#, Dongcheng Dist., Beijing 100730, People's Republic of China

Full list of author information is available at the end of the article

\section{Key points}

- 3D turbo spin echo amide proton transfer-weighted (APTw) imaging is feasible for detecting endometrioid endometrial adenocarcinoma (EEA).

- APTw values could differentiate mismatch repair deficiency (dMMR) from mismatch repair proficient (pMMR) tumors in EEA. 
- ADC values reveal no significant difference between $\mathrm{dMMR}$ and $\mathrm{pMMR}$ group.

\section{Introduction}

Endometrial carcinoma (EC) is the seventh most common malignancy worldwide and the only gynecological cancer with a rising incidence and mortality rate [1]. A novel molecular classification that accurately reflects underlying tumor biology and clinical outcomes with potential targeted and immuno-oncology treatment strategies for different subgroups was recently recommended for all patients with EC. Immunohistochemical markers, including mismatch repair (MMR) proteins (MLH1, MSH2, MSH6, and PMS2) serve as key components in this molecular classification [2, 3]. Microsatellite instabilityhigh (MSI-H) constitutes an MMR deficiency (dMMR) phenotype that is present in $20-30 \%$ of EC patients and leads to the accumulation of high mutational loads $[4,5]$. Tumors without dMMR/MSI-H are considered MMR proficient (pMMR)/microsatellite stable (MSS) [6, 7]. An estimated $3-5 \%$ of all EC patients are concomitant with hereditary nonpolyposis colorectal cancer which is also known as Lynch syndrome [8]. dMMR is detected in more than $90 \%$ of colonic and endometrial tumors in patients with Lynch syndrome [9]. MMR immunohistochemistry should be performed for the pre-screening of Lynch syndrome in all ECs irrespective of histologic subtype [2].

Magnetic resonance imaging (MRI) plays an essential role in the multidisciplinary management of EC, including characterization and staging of neoplasms, treatment decision-making, and subsequent follow-up [10]. Of note, diffusion weighted imaging (DWI), measuring water molecular mobility, has added considerable value to anatomical imaging and facilitated diagnosis [11]. Bhosale et al. reported the use of intravoxel incoherent motion (IVIM)-derived parameters (ADC values and true diffusion coefficient $\left[D_{t}\right]$ values) to determine microsatellite (MSI) status in 12 patients with Federation of Gynecology and Obstetrics (FIGO) stage I EC. Nevertheless, these methods may result in conflicting results or overlap in the measured values [12]. The argument that DWI models lack elements for characterizing biological phenomena and the remaining unresolved parameters derived from complex models present current challenges for application of these approaches in diagnosis [13]. As such, the deployment and evaluation of novel functional MRI techniques that provide new perspectives for analysis of MSI ECs is required.

Amide proton transfer-weighted (APTw) MRI was first introduced by Zhou et al. in 2003 [14]. It is a novel contrast-agent-free MRI technique that addresses the need for endogenous molecular imaging in oncology [15] and has proven valuable for the staging and characterization of tumors [16-18]. As a form of chemical exchange saturation transfer (CEST) imaging, APTw MRI is based on frequent exchange between amide protons in small proteins/peptides and the surrounding water protons, and the transfer of nuclear spin saturation from amide protons to water protons [19] results in water proton signal reduction. Studies have reported that APTw values correlate with cell proliferation and can be used as biomarkers of tumor malignancy $[14,15,20]$. APTw MRI has been predominantly utilized for the central nervous system, and its clinical utility extends to diagnosing tumors of the head and neck, breast, lung, prostate, and rectal cancers [21-26]. The use of two-dimensional (2D) APTw imaging for endometrioid endometrial adenocarcinoma (EEA) was first reported by Yukihisa et al. in 2018. In this seminal report, APTw signal intensities were significantly higher for grade 3 EEA than for grade 1 EEA [27]. Recently, this imaging marker was proven valuable in estimating histological features and risk stratification in early-stage EC [28]. In 2021, Li et al. first reported the feasibility of using three-dimensional (3D) APTw imaging for identifying endometrial adenocarcinoma, that its APTw values were significantly higher than that of benign uterine lesions, and also found APTw values exhibited a moderate positive correlation with Ki-67 proliferation status [29, 30]. The purpose of this study was to investigate the utility of 3D APTw imaging for differentiating between $\mathrm{MMMR}$ and pMMR tumors in EEA. This study may provide insight into potential application of APTw imaging in identifying MMR status in EEA.

\section{Materials and methods Study population}

This study was approved by the Institutional Review Board and complied with ethical committee standards. Written informed consent was obtained from all participants. The sample size was estimated by considering the difference in APTw values between the dMMR and pMMR groups as the primary outcome. The error was set at 0.05 , and the power level was set to $80 \%$. Based on data from the pathology department, a standard deviation of 0.4 was expected and a proportion of 0.3 for the $\mathrm{dMMR}$ group was proposed. Therefore, a total sample size of 30 was estimated using the G*Power 3 (version 3.1.9.7) sample size calculation program. A higher number of patients was enrolled in consideration of potential dropouts and poor image quality.

From January 2018 to October 2020, 78 consecutive female patients aged 25-78 years (mean age, $47.0 \pm 14.9$ years) with suspicious endometrial lesions and without MR scanning contraindications were prospectively enrolled in this pelvic APTw MRI study. Twenty-six patients without postoperative pathological diagnosis were excluded. Fifty-two patients who 
underwent staging surgery within 2 weeks after MR scanning were included for further analysis (Fig. 1).

\section{MRI}

An empty bladder was needed. $10 \mathrm{~mL}$ of glycerin enema was administered into the rectum 30 min before pelvic MRI examination to reduce air in the rectum and sigmoid. No pre-medications were used to control uterine peristalsis. Pelvic scans were performed with a clinical whole-body 3.0-T MRI unit (Ingenia 3.0 T; Philips Healthcare, Best, the Netherlands). A 16-channel dS Torso coil, which enabled parallel imaging with embedded coils, was applied above the patients. T2-weighted, T1-weighted, and DWI sequences were obtained following with an APTw sequence. A detailed overview of the
MRI parameters is listed in Table 1. An ADC map was generated by referring to the signal intensities of DWI with $b$ values of 0 and $1000 \mathrm{~s} / \mathrm{mm}^{2}$.

A 3D APTw imaging sequence was used in this study, with long radiofrequency (RF) pulses at $2-\mu \mathrm{T}$ amplitude applied to saturate the amide proton spin signals. In order to generate a continuous 2-s long saturation RF, two RF transmit coils were used and each was turned on for 500-ms alternatively to last four sections. To reduce CEST artifacts from the presence of fat in the pelvis, an asymmetric frequency-modulated pulse (chemical-shiftselective) was applied to suppress fatty tissue MR signals. The APTw sequence was repeated nine times at seven RF saturation offsets (for convenience, the water resonance frequency was set at $0 \mathrm{ppm}$ in the $\mathrm{z}$-spectrum for

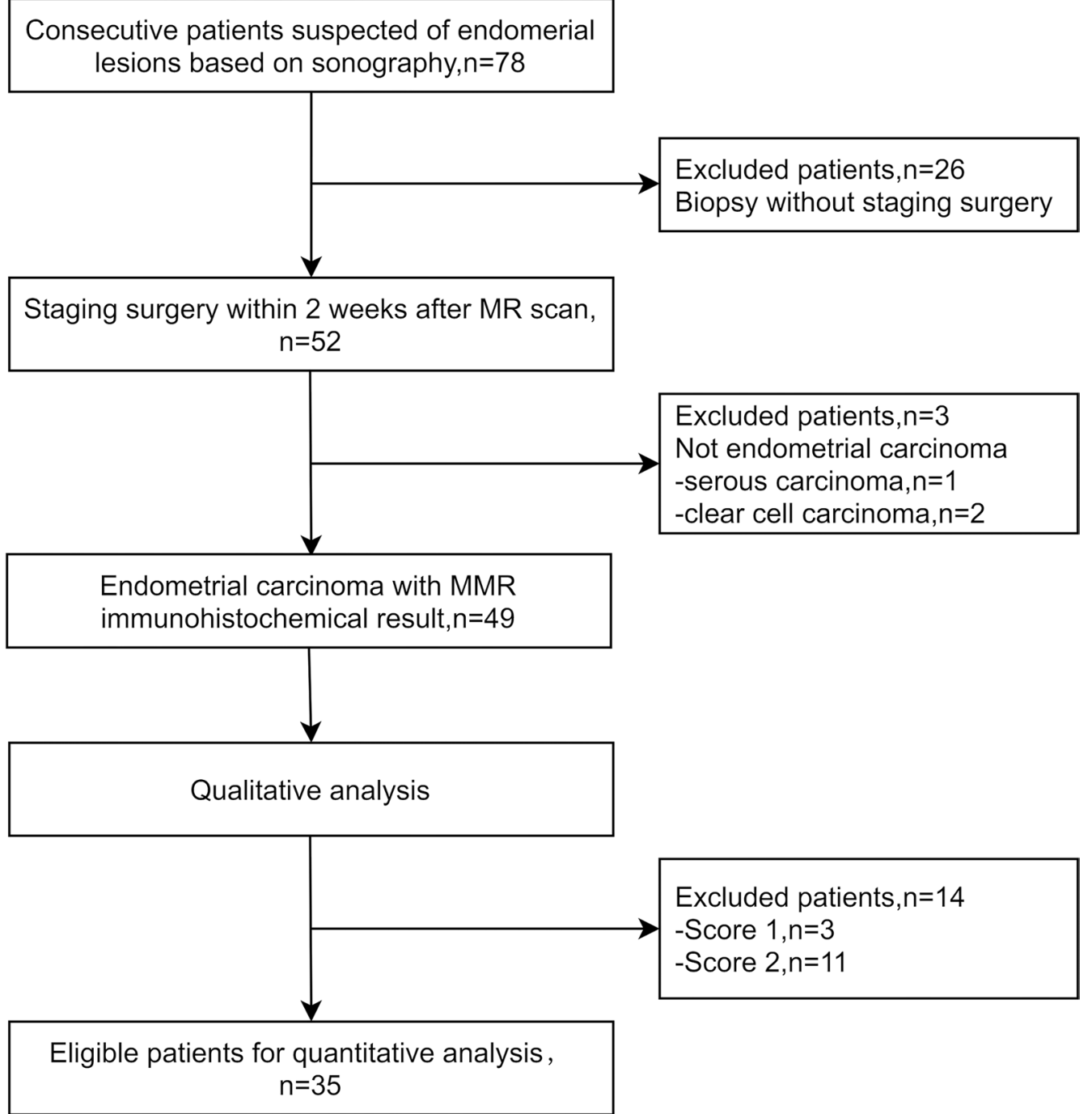

Fig. 1 Flow chart of the study cohort. MR= magnetic resonance; $M M R=$ mismatch repair 
Table 1 MR imaging parameters details

\begin{tabular}{|c|c|c|c|c|c|}
\hline Parameters & APTw & T1-weighted & T2-weighted & T2-weighted & Diffusion-weighted \\
\hline Imaging acquisition & 3DTSE & TSE & TSE & TSE & EPI \\
\hline Orientation & Axial & Axial & Axial & Sagittal & Axial \\
\hline Repetition time/echo time (ms) & $7188 / 5.4$ & $507 / 8.0$ & $3471 / 100$ & $3500 / 100$ & $4656 / 82$ \\
\hline Flip angle $\left(^{\circ}\right)$ & 90 & 90 & 90 & 90 & 90 \\
\hline Field of view $\left(\mathrm{mm}^{2}\right)$ & $300 \times 243$ & $240 \times 300$ & $400 \times 400$ & $260 \times 260$ & $300 \times 218$ \\
\hline Matrix (frequency $\times$ phase) & $120 \times 96$ & $320 \times 299$ & $400 \times 400$ & $512 \times 512$ & $100 \times 72$ \\
\hline Spatial resolution $\left(\mathrm{mm}^{2}\right)$ & $2.5 \times 2.5$ & $0.75 \times 1.0$ & $1.0 \times 1.0$ & $0.51 \times 0.51$ & $3.0 \times 3.0$ \\
\hline Slice thickness (mm) & 5 & 4 & 3 & 3 & 4 \\
\hline Slice gap (mm) & 0 & 1 & 0.3 & 0.3 & 1 \\
\hline No. of slices & 9 & 40 & 41 & 29 & 25 \\
\hline ETL & 158 & 4 & 24 & 32 & $\ldots$ \\
\hline EPI factor & $\ldots$ & $\ldots$ & $\ldots$ & $\ldots$ & 55 \\
\hline SENSE factor & 2 & 1.6 & 3.5 & 1.5 & 1.8 \\
\hline$b$ values & $\ldots$ & $\ldots$ & $\ldots$ & $\ldots$ & 0,1000 \\
\hline Fat suppression & SPIR & $\ldots$ & $\ldots$ & $\ldots$ & SPAIR \\
\hline Total image time (min:s) & $7: 33$ & $3: 18$ & $2: 36$ & $3: 21$ & $1: 30$ \\
\hline
\end{tabular}

$M R=$ Magnetic resonance; $A P T w=$ Amide proton transfer-weighted; $T S E=$ Turbo spin echo; $E T L=E c h o$ train length; $E P I=E c h o ~ p l a n a r$ imaging; $S E N S E=S e n s i t i v i t y$ encoding; SPAIR = Spectral attenuation with inversion recovery; SPIR = Spectral presaturation with inversion recovery. Other key parameters for APTw imaging include 2 -s long radiofrequency (RF) pulses at $2-\mu \mathrm{T}$ power, and 9 acquisitions including 7 different frequency saturation offsets $(-4.3,-3.5,-2.7,2.7,3.5,4.3$ and $-1560 \mathrm{ppm}$ ) and 2 additional acquisitions with echo shifts

APT-related offset definitions). Saturation RF pre-pulses were applied selectively at $3.5 \mathrm{ppm}$ which is proven to be the amide proton frequency offset [14]. The APT effect is proportional to the difference of MRI signals with and without saturation pulses at $3.5 \mathrm{ppm}$. However, the RF magnetic transfer effects have to be compensated for APTw quantification, so the APTw signal is defined as Magnetization Transfer Ratio (MTR) Asymmetry at $\pm 3.5 \mathrm{ppm}$. Moreover, B0 inhomogeneity strongly impacts the accuracy because Larmor frequencies of amide protons are shifted if B0 inhomogeneity exists [31]. Hence, two additional acquisitions with different echo shifts of $0.5 \mathrm{~ms}$ were performed with the same saturation pre-pulses, and the image volumes with three different echo times (all at offset of $+3.5 \mathrm{ppm}$ ) were used to derive the B0 field map. To compensate for B0 inhomogeneity, four more saturation frequency offsets $(3.5 \pm 0.8 \mathrm{ppm}$ and $-3.5 \pm 0.8 \mathrm{ppm})$ in $\mathrm{z}$-spectrum were measured. The $\mathrm{Z}$-spectrum was aligned for each voxel, and the signals at targeting offsets, $S(-3.5 \mathrm{ppm})$ and $S(+3.5 \mathrm{ppm})$, were calculated using Lagrange interpolation [31]. In summary, APTw values were calculated according to the following equation:

$$
\text { APT weighting }=\frac{S(-3.5 \mathrm{ppm})-S(+3.5 \mathrm{ppm})}{S(-1560 \mathrm{ppm})}
$$

where the $S(-1560 \mathrm{ppm})$ was the signal from one acquisition with saturation RF at extra-large offset $(-1560 \mathrm{ppm})$ as the control for APTw quantification. The
APTw specific absorption ratio (SAR) value was $1.1 \mathrm{~W} /$ $\mathrm{kg}$, which fell within the U.S. Food and Drug Administration guidelines. The middle slice of the APTw images was identified based on the largest cross-section of the lesions present on conventional MR images selected by radiologists with 10-17 years of experience in interpreting MR images of the female pelvis.

\section{APTw image quality analysis}

The APTw calculations, including z-spectrum shift and interpolation, were performed online using a MR control console. Raw image datasets were transferred to a workstation (Intellispace Portal; Version 10.1.0.64190; Philips Healthcare, Best, the Netherlands) for post-processing. All MR images were reviewed by three radiologists (Xue HD, He YL, and Lin CY; observers 1, 2, and 3 with 17, 10 , and 5 years of experience in interpreting pelvic MR images, respectively), who had previously evaluated over 300 APTw images and were blinded to the patients' clinical and histopathologic data. Based on the APTw image quality evaluation criteria for uterine cervical cancer [32], the three observers independently ranked the APTw images relative to image quality and measurement confidence on a 5-point Likert scale with respect to image blur, distortion, motion and ghosting artifacts, lesion recognition, and contour delineation. Table 2 summarizes the marking scale and the APTw image scores assessed by the three readers. 
Table 2 Image quality evaluation of APTw images

\begin{tabular}{|c|c|c|c|c|}
\hline Image scores & Scale of marks & Reader 1 & Reader 2 & Reader 3 \\
\hline 5 & Good image quality with tumor detectable and lesion contour clearly delineated on APTw images & $n=6$ & $n=5$ & $n=6$ \\
\hline 4 & $\begin{array}{l}\text { Tumor lesion could be recognized on APTw images, but contour was not so well delineated, refer- } \\
\text { ence information on conventional MR images needed for region of interest (ROI) analysis }\end{array}$ & $n=17$ & $n=20$ & $n=18$ \\
\hline 3 & Tumor undetectable without reference to conventional MR images & $n=12$ & $n=10$ & $n=11$ \\
\hline 2 & $\begin{array}{l}\text { Poor APTw image quality with obvious artifacts, although the tumor lesion was revealed on con- } \\
\text { ventional MR images }\end{array}$ & $n=12$ & $n=11$ & $n=10$ \\
\hline 1 & No lesions were identified on APTw or any conventional MR images & $n=2$ & $n=3$ & $n=4$ \\
\hline
\end{tabular}

APTw amide proton transfer-weighted

\section{APTw and ADC value measurements}

Two observers (Xue HD and He YL; observers 1 and 2) independently measured the APTw values with image quality scores of no less than 3 . With reference to conventional $M R$ images, the observers selected a single APTw image and ADC map slice with the maximum lesion area and drew a smooth contour region of interest (ROI) to cover the lesion. The mean APTw and ADC values in areas of the ROIs were recorded.

\section{Histopathologic and MMR immunohistochemistry analysis} Surgically resected specimens stained with hematoxylin and eosin, and MMR immunohistochemical staining were reviewed by a pathologist (Chen B with 10 years of experience in gynecological pathology), who was blinded to the clinical and imaging data. The aggressiveness of each EEA specimen was categorized into three groups based on the FIGO grading system criteria: grade 1, welldifferentiated EEA; grade 2, moderately differentiated EEA; and grade 3, poorly differentiated EEA [33]. The expression of MMR proteins MLH1, MSH2, MSH6, and PMS2 was detected by immunohistochemistry using an FFPE tissue microarray and a Ventana Benchmark XT autostainer (Ventana Medical Systems Inc., Tucson, AZ) according to the manufacturer's protocols. The absence of nuclear staining in tumor cells was considered a "loss of expression" with intervening stromal positivity serving as an internal control. The complete expression of all four MMR proteins was considered a case of pMMR. The loss of at least one MMR protein was considered a case of dMMR [34].

\section{Statistical analysis}

Statistical analysis was performed using standard statistical software (Prism 8, GraphPad Software, San Diego, CA; SPSS Statistics 23, IBM, NY, USA). For image quality assessment, Kendall's $W$ test was used to evaluate the inter-observer agreement. Inter-class correlation coefficients (ICCs) were computed to evaluate the inter-observer agreement of the APTw value measurements. Kendall's $W$ and ICC values of less than 0.4, $0.41-0.75$, and greater than 0.75 , were considered to indicate positive but poor, good, and excellent agreement, respectively. The Shapiro-Wilk test was performed to evaluate the normality of the distribution of APTw and $\mathrm{ADC}$ values. APTw and ADC values are presented as the mean \pm standard deviation. For normally distributed data, Student's t-test was performed to compare APTw and ADC values between the $\mathrm{AMMR}$ and pMMR groups. APTw and ADC values were compared among the three grades using a one-way analysis of variance with Scheffe's post hoc test. Statistical significance was set at $p<0.05$. Receiver operating characteristic (ROC) analysis was performed to determine the feasible threshold value with assessment of sensitivity and specificity.

\section{Results}

A flowchart of the study population is presented in Fig. 1. Three patients with other histological types of EC (serous carcinoma, $n=1$ and clear cell carcinoma, $n=2$ ) were excluded. Thus, 49 patients with pathological confirmation of EEA were included in the analysis. MR and immunohistochemical staining images of $\mathrm{dMMR}$ and pMMR are presented in Figs. 2 and 3.

(See figure on next page.)

Fig. 2 A 56-year-old woman with post-menopause uterus bleeding and mismatch repair deficient (dMMR) endometrioid endometrial adenocarcinoma (EEA), grade 3 with stage IA. MR images of: a T2WI; b APTw, with mean APTw value $3.0 \%$ by two readers; $\mathbf{c}$ DWI original map $\left(b=1000 \mathrm{~s} / \mathrm{mm}^{2}\right)$; d pseudo colored map of ADC, with mean ADC values $0.721 \times 10^{-3} \mathrm{~mm}^{2} / \mathrm{s}$ by two readers. MMR immunohistochemistry analysis revealed the loss of nuclear staining in tumor cells for MLH1 (e) and PMS2 (f), respectively ( $\times 100$ magnification); positive staining in stromal cells and normal endometrial glands serves as internal control. Loss of MSH2 (g) and MSH6 (h) expression, respectively ( 100 magnification). Brown stain is positive staining and blue counterstain is indicative of absent or negative staining 

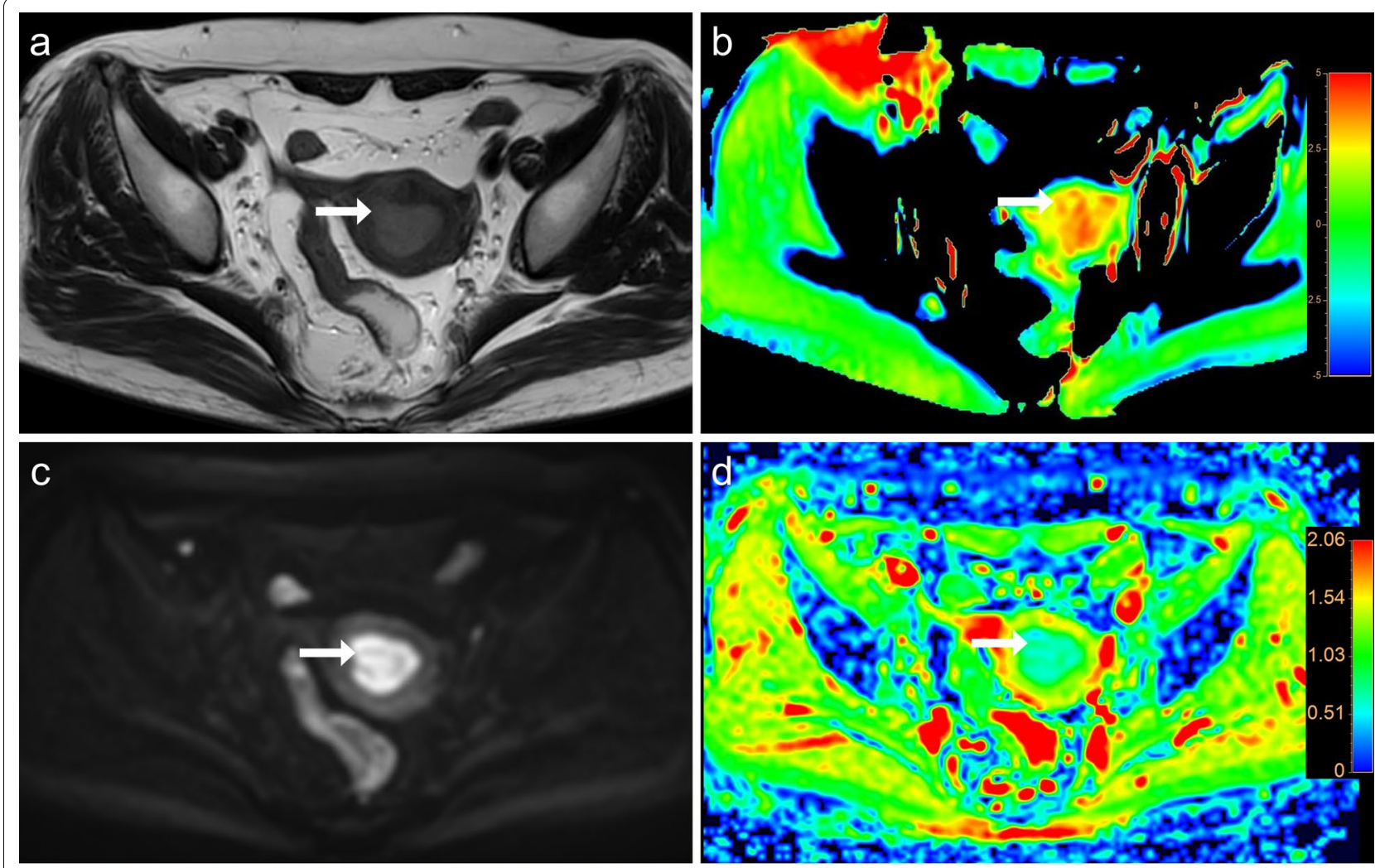

e

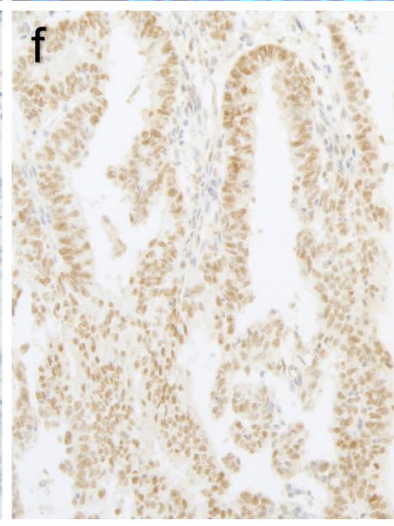

g

h

Fig. 2 (See legend on previous page.) 

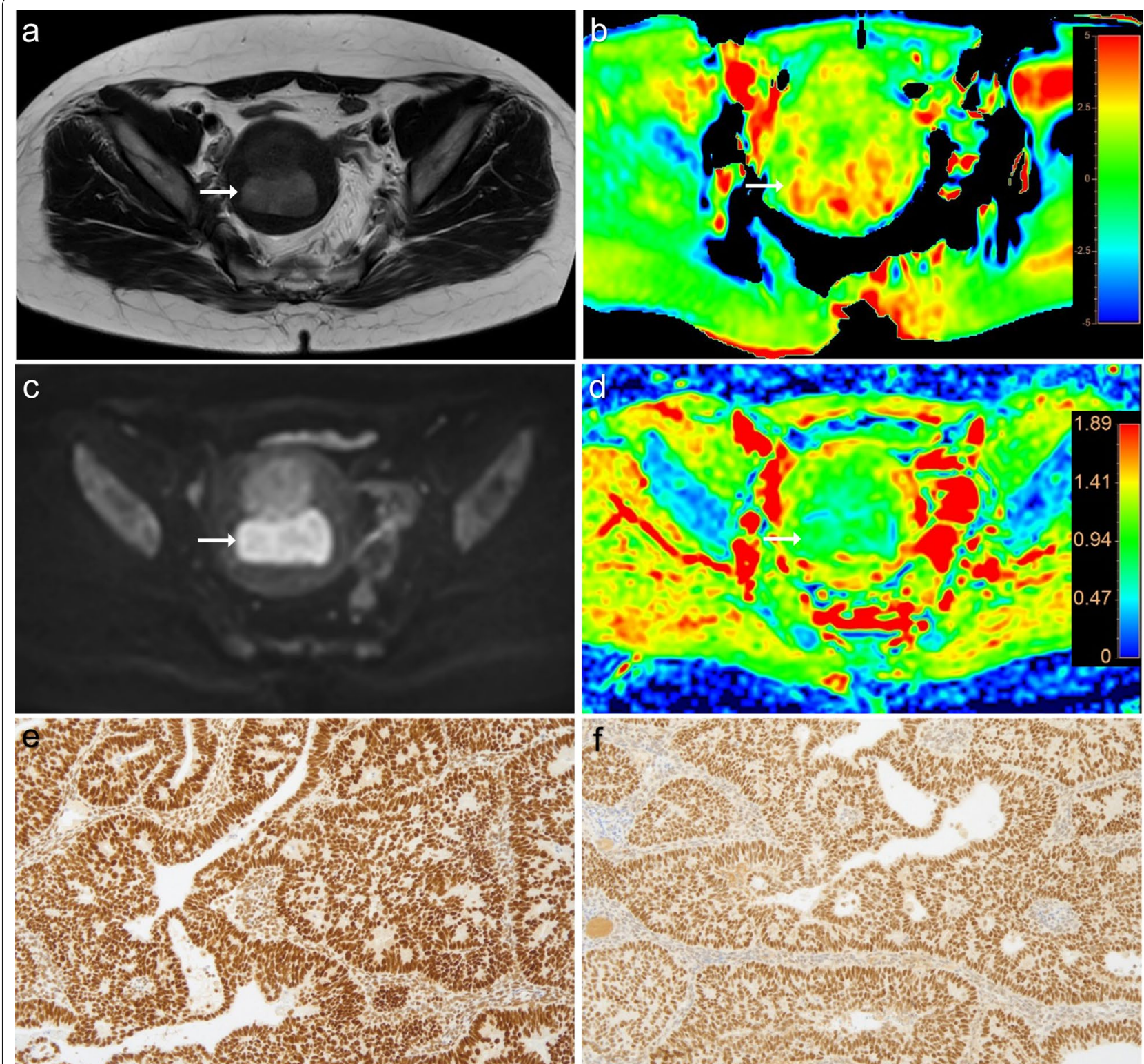

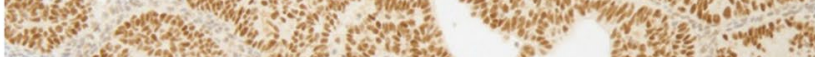

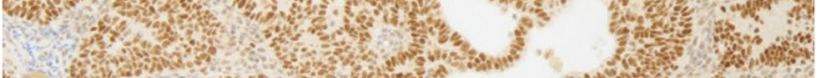

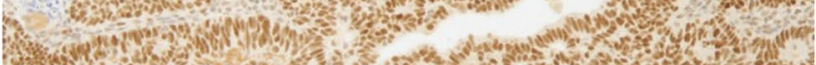

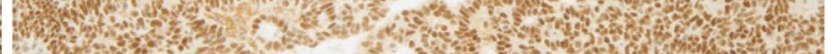
(1)

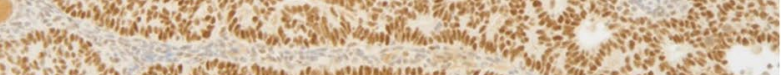

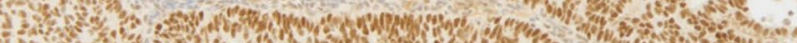

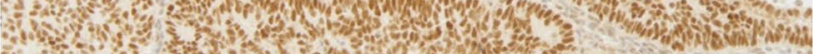

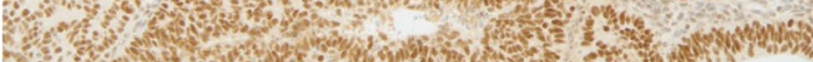

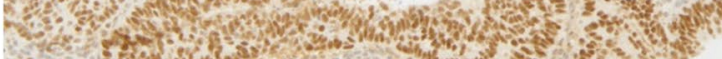
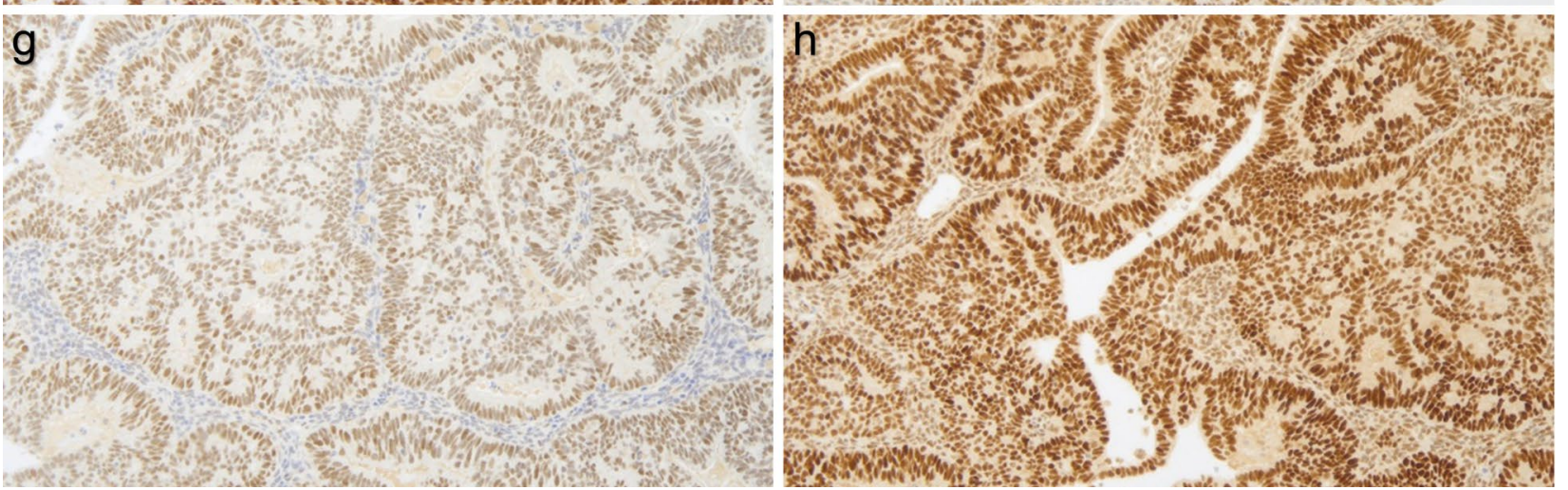

Fig. 3 A 53-year-old woman with irregular menstruation and mismatch repair proficient (pMMR) EEA, grade 2 with stage IA. MR images of: (a) T2WI; b APTw, with mean APTw value $2.8 \%$ by two readers; c DWI original map $\left(b=1000 \mathrm{~s} / \mathrm{mm}^{2}\right)$; $\mathbf{d}$ pseudo colored map of ADC, with mean ADC values $0.763 \times 10^{-3} \mathrm{~mm}^{2} / \mathrm{s}$ by two readers. MMR immunohistochemistry analysis $(\mathbf{e}-\mathbf{h})$ revealed the intact nuclear staining for mismatch repair proteins MLH1, PMS2, MSH2 and MSH6 in tumor cells, respectively ( $\times 100$ magnification) 


\section{APTw image quality analysis}

In total, 49 patients with EEA aged 26-77 years (mean age, 50.9 years) were enrolled for evaluation of APTw image quality. Table 2 summarizes the APTw image scores assessed by the three observers, which exhibited excellent agreement (Kendall's $W=0.867, p<0.001$ ). Most cases were ranked with a score of 4 . Poor image quality was observed in $28.6 \%$ of the cases due to distortion and artifacts.

\section{APTw and ADC value measurements}

APTw values were obtained in 35 cases of EEA aged 26-76 years (mean age, 50.0 years). Patient demographics are presented in Table 3 . The mean ROI area was $315.7(21.1-1288.0) \mathrm{mm}^{2}$. The APTw values were normally distributed $(p=0.890)$. The mean APTw value was $2.9 \pm 0.5 \%$ with an inter-observer ICC of 0.985 (95\% confidence interval [CI]: 0.971-0.993).

For ADC value measurements, the mean ROI area of endometrial lesions was $300.2(32.3-1021) \mathrm{mm}^{2}$. ADC values were normally distributed $(p=0.335)$. The mean ADC value was $0.895 \pm 0.101 \times 10^{-3} \mathrm{~mm}^{2} / \mathrm{s}$ with an inter-observer ICC of 0.976 (95\% CI: 0.954-0.988).

Table 3 Characteristics of the 35 patients with EEA

\begin{tabular}{ll}
\hline Parameter & No. of patients \\
\hline Mean age (age range) & 50.0 (26-77) \\
FIGO stage & \\
IA & 29 \\
IB & 2 \\
IIIA & 1 \\
IIIC2 & 2 \\
IVB & 1 \\
MMR status & \\
dMMR & 9 \\
IA & 5 \\
IB & 2 \\
IIIC2 & 1 \\
IVB & 1 \\
pMMR & 26 \\
IA & 24 \\
IIIA & 1 \\
IIIC2 & 1 \\
Histologic grade & \\
Grade 1 & 17 \\
Grade 2 & 15 \\
Grade 3 & 3 \\
\hline
\end{tabular}

EEA endometrioid endometrial adenocarcinoma, FIGO Federation of Gynecology and Obstetrics

\section{Comparison of APTw and ADC values between dMMR and $\mathrm{pMMR}$ groups}

The $\mathrm{dMMR}$ and pMMR groups comprised 9 and 26 cases, respectively. The mean APTw value was significantly higher in the $\mathrm{dMMR}$ group than in the pMMR group $(3.2 \pm 0.3 \%$ and $2.8 \pm 0.5 \%$, respectively; $p=0.019$; Fig. 4 ). The area under the curve of ROC analysis for differentiating the dMMR and pMMR groups was 0.778 (Fig. 5). The feasible threshold value was determined to be $3.0 \%$, with a sensitivity of $88.9 \%$ and specificity of $69.2 \%$. No significant differences were observed in mean $\mathrm{ADC}$ values between the dMMR group $\left(0.874 \pm 0.104 \times 10^{-3} \mathrm{~mm}^{2} / \mathrm{s}\right)$ and $\mathrm{pMMR}$ group $\left(0.903 \pm 0.100 \times 10^{-3} \mathrm{~mm}^{2} / \mathrm{s} ; p=0.476\right.$, Fig. 4).

\section{Comparison of APTw and ADC values between histologic grades}

The mean APTw values of grade 1 , grade 2 , and grade 3 were $2.9 \pm 0.5 \%, 2.9 \pm 0.4 \%$ and $3.1 \pm 0.6 \%$, respectively; the mean ADC values of grade 1 , grade 2 and grade 3 were $0.922 \pm 0.119 \times 10^{-3} \mathrm{~mm}^{2} / \mathrm{s}, \quad 0.876 \pm 0.070 \times 10^{-3}$ $\mathrm{mm}^{2} / \mathrm{s}$ and $0.845 \pm 0.110 \times 10^{-3} \mathrm{~mm}^{2} / \mathrm{s}$, respectively (Fig. 4). No significant differences in APTw or ADC values were observed among the three histologic grades ( $p=0.766$ and $p=0.295$, respectively).

\section{Discussion}

In this study, we examined the utility of 3D APTw MRI for distinguishing $\mathrm{AMMR}$ and $\mathrm{PMMR}$ in EEA. Our findings indicate that $3 \mathrm{D}$ TSE APTw imaging is a feasible approach for detecting EEA and that APTw values have the potential to differentiate $\mathrm{dMMR}$ from pMMR tumors in EEA.

In our cohort, $25.7 \%(9 / 35)$ of patients with EEA presented with $\mathrm{dMMR}$, which was consistent with the literature [35]. The relationship between MSI and prognosis in patients with EC has not been conclusively demonstrated [12]. It is reported that $\mathrm{dMMR}$ is a potential biomarker for good responders to PD-L1/PD-1 immunotherapy in EC [36]. MMR immunohistochemistry was recommended to identify MMR status for EC patients in the clinical practice. An invasive endometrial biopsy must be performed for the diagnosis. Nevertheless, it may have $10 \%$ false negatives due to the multifocal nature of EC lesions [37] and the specimens obtained are sometimes not sufficient to determine MMR status. As known, MRI plays an essential role in the preoperative evaluation of EC, which is highly specific in the assessment of the depth of myometrial invasion, cervical stromal involvement, and lymph node metastasis [38]. Moreover, MRI must be performed to assess the extension of the disease for patients considering fertility preservation treatments [39]. Multiple imaging techniques are currently being 

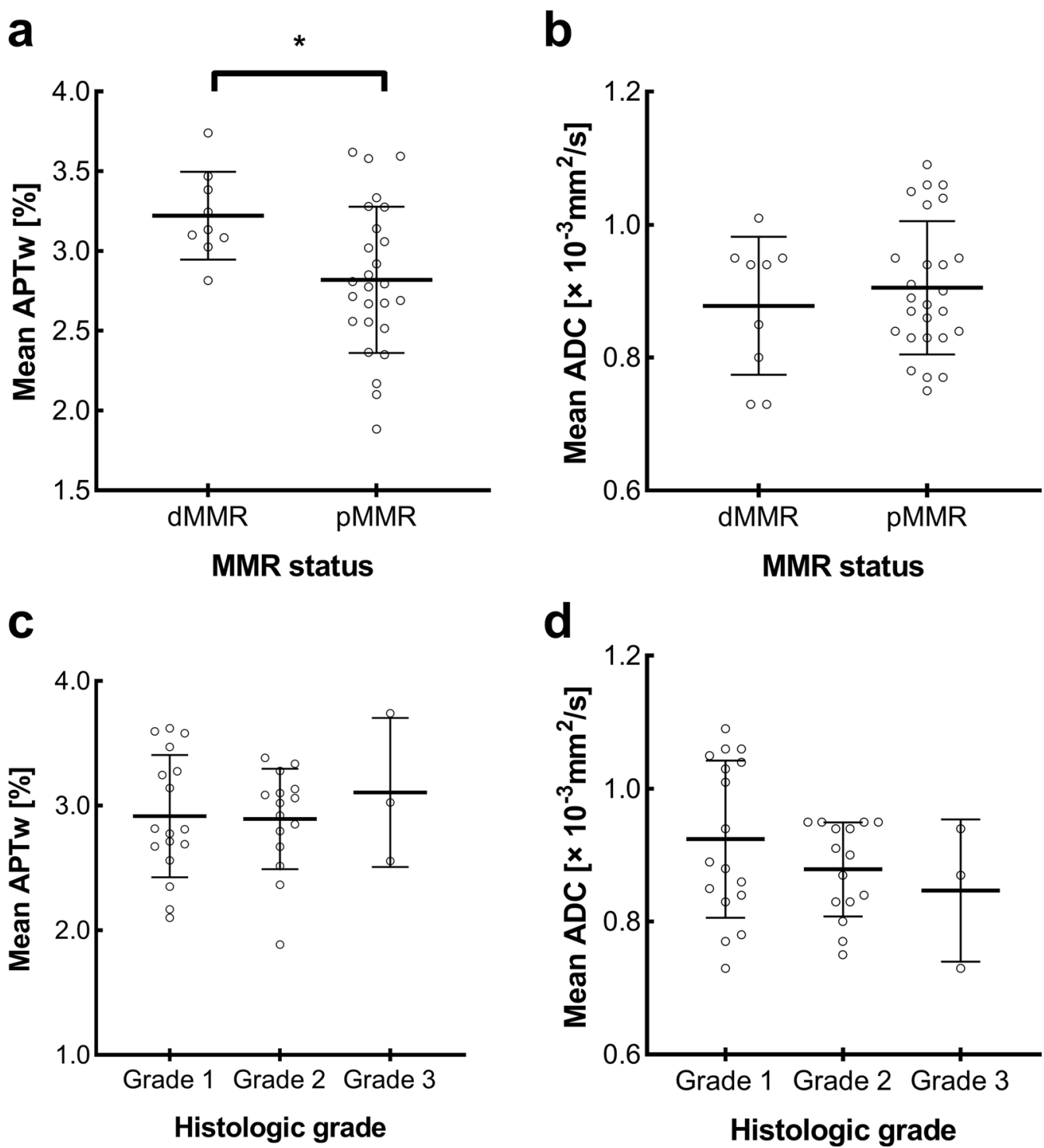

Fig. 4 Plots showing individual data points (circles), averages (transverse lines), and standard deviations (vertical lines) of (a) mean APTw values and (b) mean ADC values of dMMR and pMMR EEAs; c mean APTw values and (d) mean ADC values of three histologic grades. Individual points were averages of values calculated by two readers. $*=$ Statistically significant difference at $p<0.05$

developed to investigate tumor expression of immunotherapy targets PD-L1/PD-1. Based on conventional MR images, dMMR EC tended to be located lower in the uterus $(p=0.0366)$, although most other parameters were not significantly different to those of pMMR $\mathrm{EC}$, including the size $(p=0.97)$, spread $(p>0.99)$, and shape $(p=0.76)$ [40]. Mean ADC values exhibited a trend to be lower in dMMR EC than in PMMR EC, although this finding was not statistically significant $(p=0.15)$ [40], with similar results observed in our study. We demonstrated a correlation between pretreatment APTw values and MMR status. APT technology may enable non-invasive detection of multicellular components of tumor microenvironments to potentially predict response to immunotherapy. Additionally, it might further serve as the non-invasive screening and auxiliary differentiation of EC related to Lynch syndrome. Close surveillance in patients with EC who have dMMR and are subsequently diagnosed with Lynch syndrome is imperative to enable early detection, prevention, and treatment of other cancers [41].

Advanced APTw method was applied in this research, such as 3D acquisition for volumetric imaging, B0 inhomogeneity correction and the long saturation pulses (2-s). 


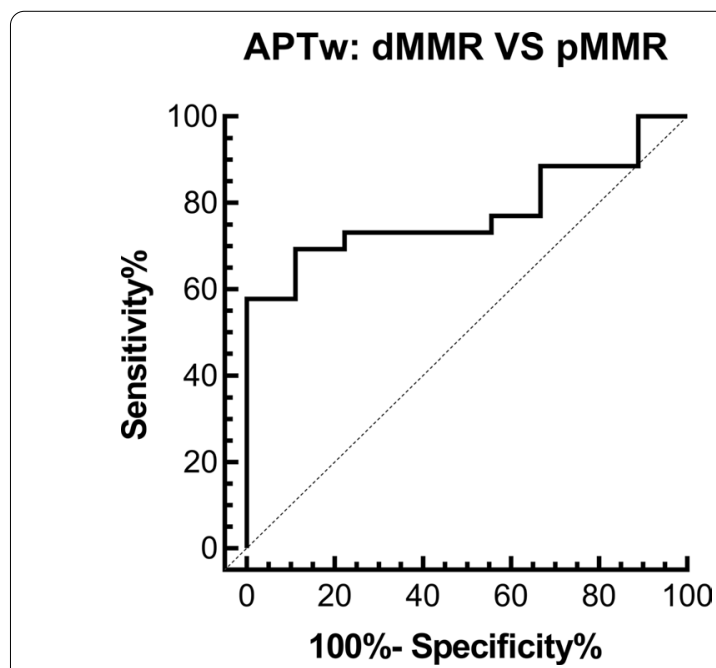

Fig. 5 Curves showing mean APTw values by using receiver operating characteristic analysis for differentiating dMMR from pMMR tumors in EEA. The area under the curve (AUC) was 0.778. The feasible threshold values were determined as $3.0 \%$ with a sensitivity of $88.9 \%$ and specificity of $69.2 \%$

Nevertheless, there are some technical challenges. First, APTw value is not necessarily be proportional to cellular proliferation, because it represents not only the concentration of mobile proteins/peptides but also chemical factors such as $\mathrm{pH}$. Secondly, the acquisition resolution of the current APTw imaging method was low; and the strong filtering in post processing resulted in even lower resolution appearance. Thirdly, though B0 field inhomogeneity correction has been applied, field inhomogeneity was still identified as a major challenge in pelvis APTw imaging. Extreme hyper/hypo intensity $(>5 \%$ and $<-5 \%)$ are presented near thighbone and hipbone, similar to hyperintensity artifacts formed around the skull in brain APTw imaging. We suspect that the Lagrange interpolation algorithm does not work well in the extrapolation conditions with large B0 field deviation. Air in digestive tracts also leads to substantial B0 field distortion, which moves during scanning and causes artifacts that hard to identify, hence administration of glycerin enema was necessary for this examination to reduce air in the rectum and sigmoid. In addition, current APTw application is longer than 5 min. Essentially, it is a 3D TSE sequence with 9 acquisitions; and there is a 2-s saturation pulse within each TR. Less acquisitions reduces acquisition time; however, the resulting image quality is poor. Shorter saturation pulse could also shorten scan duration, but the APT weighting and contrast is reduced. Lastly, we found lower APTw signal in the region closer to the cable of Torso Coil. The coil cable contained residual eddy currents and acted as an unexpected antenna that led to B1 changes during the saturation RF, as a result, it may impact APTw values' accuracy. Because of these challenges in image artifacts, we spent efforts in evaluating image qualities and exclude APTw images with artifacts in tumor region for further analysis in this study.

There are a few limitations to this study. First, this study was a single-center analysis, which may lead to selection bias. Besides, sample size was small, despite we enrolled adequate patients beyond the required sample size as mentioned above. Future large prospective studies are needed to confirm our findings. Second, although nine APTw image slices were obtained, the APTw values were measured using the single largest area to avoid unnecessary artifacts at the edge of tumors, based on other pelvic APTw imaging studies. Future studies should investigate the APTw values of the whole tumor volume and histograms. Third, the spatial resolution of the APTw imaging was $2.5 \times 2.5 \mathrm{~mm}^{2}$. As such, some small lesions with tumor areas less than $20 \mathrm{~mm}^{2}$ were difficult to evaluate and were excluded from the quantitative analysis. Improvements in the pelvic APTw sequence, such as increasing the spatial resolution, are warranted.

\section{Conclusion}

In conclusion, APTw imaging is a feasible technique for detecting MMR status in EEA. APTw values may be used as potential imaging markers to differentiate $\mathrm{dMMR}$ from pMMR tumors in EEA.

\section{Abbreviations}

2D: Two-dimensional; 3D: Three-dimensional; ADC: Apparent diffusion coefficient; APTw: Amide proton transfer-weighted; AUC: Area under the curve; CEST: Chemical exchange saturation transfer; dMMR: Mismatch repair deficiency; DWI: Diffusion-weighted imaging; EC: Endometrial carcinoma; EEA: Endometrioid endometrial adenocarcinoma; FIGO: Federation of Gynecology and Obstetrics; ICC: Inter-class correlation coefficient; MMR: Mismatch repair; MRI: Magnetic resonance imaging; MSI: Microsatellite instability; MTR: Magnetization transfer ratio; pMMR: Mismatch repair proficient; RF: Radiofrequency; ROC: Receiver operating characteristic; ROI: Region of interest; SAR: Specific absorption rate; TSE: Turbo spin echo.

\section{Acknowledgements}

The authors would like to thank Dr. Yuming Chong and Dr. Chang Han for their supports.

\section{Authors' contributions}

Guarantor of Integrity of the Entire Study: HYL. Study Concepts: HYL, LY, L XY. Data Acquisition: H YL, LY, L CY, Q YF, Z HL, W QL, RJ, ZJ, CB, Y JJ, XY. Quality Control of Data: LY, L XY, L CY. Data Analysis and Interpretations: $H Y L, L C Y, L$ $X Y, X$ HD. Statistical Analysis: LXY. Manuscript Preparation: H YL, L XY, LY, W XQ. Manuscript Editing and Reviewing: $H Y L, J Z Y, X H D, Y J J, X Y$. All authors read and approved the final manuscript.

\section{Funding}

This work was supported by grants from Natural Science Foundation of China (Grant No. 81901829) and the Fundamental Research Funds for the Central Universities (Grant No. 3332019032). 


\section{Availability of data and materials}

Study data can be made available upon documented request.

\section{Declarations}

\section{Ethics approval and consent to participate}

Local ethics committee has approved the project and all participants signed informed consent.

\section{Consent for publication}

Informed consent was obtained from all individual participants included in the study.

\section{Competing interests}

Co-Author Xiaoqi Wang is employee of Philips Healthcare China. The other authors have no conflicts of interest to disclose. The authors who are not employed by Philips were in control of this study.

\section{Author details}

${ }^{1}$ Department of Obstetrics and Gynecology, Peking Union Medical College Hospital, Peking Union Medical College and Chinese Academy of Medical Sciences, National Clinical Research Center for Obstetric and Gynecologic Diseases, Beijing, People's Republic of China. ${ }^{2}$ Department of Radiology, Peking Union Medical College Hospital, Peking Union Medical College and Chinese Academy of Medical Sciences, Shuai Fu Yuan 1\#, Dongcheng Dist., Beijing 100730, People's Republic of China. ${ }^{3}$ Philips Healthcare China, Beijing, People's Republic of China. ${ }^{4}$ Department of Pathology, Peking Union Medical College Hospital, Peking Union Medical College and Chinese Academy of Medical Sciences, Beijing, People's Republic of China.

Received: 28 September 2021 Accepted: 2 November 2021 Published online: 11 December 2021

\section{References}

1. Siegel RL, Miller KD, Jemal A (2020) Cancer statistics, 2020. CA Cancer J Clin 70(1):145-164

2. Concin N, Matias-Guiu X, Vergote I, et al. (2021) ESGO/ESTRO/ESP guidelines for the management of patients with endometrial carcinoma. Radiother Oncol 154:327-353

3. Vermij L, Smit V, Nout R, Bosse T (2020) Incorporation of molecular characteristics into endometrial cancer management. Histopathology 76(1):52-63

4. Matthews KS, Estes JM, Conner MG, et al. (2008) Lynch syndrome in women less than 50 years of age with endometrial cancer. Obstet Gynecol 111(5):1161-1166

5. Bonneville R, Krook MA, Kautto EA, et al. (2017) Landscape of microsatellite instability across 39 cancer types. JCO Precis Oncol 1:1-15

6. Richman S (2015) Deficient mismatch repair: read all about it (Review). Int J Oncol 47(4):1189-1202

7. Camp MC, Wong WW, Filip Z, Carter CS, Gupta SC (2011) A quantitative analysis of periorbital aging with three-dimensional surface imaging. J Plast Reconstr Aesthet Surg 64(2):148-154

8. Aarnio M, Sankila R, Pukkala E, et al. (1999) Cancer risk in mutation carriers of DNA-mismatch-repair genes. Int J Cancer 81(2):214-218

9. Loukola A, Salovaara R, Kristo P, et al. (1999) Microsatellite instability in adenomas as a marker for hereditary nonpolyposis colorectal cancer. Am J Pathol 155(6):1849-1853

10. Manfredi R, Mirk P, Maresca G, et al. (2004) Local-regional staging of endometrial carcinoma: role of MR imaging in surgical planning. Radiology 231(2):372-378

11. Nougaret S, Horta M, Sala E, et al. (2018) Endometrial cancer MRI staging: updated guidelines of the European Society of urogenital radiology. Eur Radiol 29(2):792-805

12. Bhosale P, Ramalingam $P, M a ~ J$, et al. (2017) Can reduced field-of-view diffusion sequence help assess microsatellite instability in FIGO stage 1 endometrial cancer? J Magn Reson Imaging 45(4):1216-1224

13. Wang YXJ, Huang H, Zheng C-J, Xiao B-H, Chevallier O, Wang W (2021) Diffusion-weighted MRI of the liver: challenges and some solutions for the quantification of apparent diffusion coefficient and intravoxel incoherent motion. Am J Nucl Med Mol Imaging 11(2):107-142

14. Zhou J, Lal B, Wilson DA, Laterra J, van Zijl PC (2003) Amide proton transfer (APT) contrast for imaging of brain tumors. Magn Reson Med 50(6):1120-1126

15. Kamimura K, Nakajo M, Yoneyama T, et al. (2019) Amide proton transfer imaging of tumors: theory, clinical applications, pitfalls, and future directions. Jpn J Radiol 37(2):109-116

16. Jiang S, Eberhart CG, Lim M, et al. (2019) Identifying recurrent malignant glioma after treatment using amide proton transfer-weighted $\mathrm{mr}$ imaging: a validation study with image-guided stereotactic biopsy. Clin Cancer Res 25(2):552-561

17. Zhou J, Heo H-Y, Knutsson L, van Zijl PCM, Jiang S (2019) APT-weighted MRI: techniques, current neuro applications, and challenging issues. J Magn Reson Imaging 50(2):347-364

18. Jiang S, Eberhart CG, Zhang Y, et al. (2017) Amide proton transferweighted magnetic resonance image-guided stereotactic biopsy in patients with newly diagnosed gliomas. Eur J Cancer 83:9-18

19. van Zijl PCM, Yadav NN (2011) Chemical exchange saturation transfer (CEST): what is in a name and what isn't? Magn Reson Med 65(4):927-948

20. Zhou J, Tryggestad E, Wen Z, et al. (2011) Differentiation between glioma and radiation necrosis using molecular magnetic resonance imaging of endogenous proteins and peptides. Nat Med 17(1):130-134

21. Law BKH, King AD, Ai QY, et al. (2018) Head and neck tumors: amide proton transfer MRI. Radiology 288(3):782-790

22. Su C, Zhao L, Li S, et al. (2019) Amid proton transfer (APT) and magnetization transfer (MT) MRI contrasts provide complimentary assessment of brain tumors similarly to proton magnetic resonance spectroscopy imaging (MRSI). Eur Radiol 29(3):1203-1210

23. Zimmermann F, Korzowski A, Breitling J, et al. (2020) A novel normalization for amide proton transfer CEST MRI to correct for fat signal-induced artifacts: application to human breast cancer imaging. Magn Reson Med 83(3):920-934

24. Ohno Y, Kishida Y, Seki S, et al. (2018) Amide proton transfer-weighted imaging to differentiate malignant from benign pulmonary lesions: Comparison with diffusion-weighted imaging and FDG-PET/CT. J Magn Reson Imaging 47(4):1013-1021

25. Takayama Y, Nishie A, Sugimoto M, et al. (2016) Amide proton transfer (APT) magnetic resonance imaging of prostate cancer: comparison with Gleason scores. MAGMA 29(4):671-679

26. Nishie A, Asayama Y, Ishigami K, et al. (2019) Amide proton transfer imaging to predict tumor response to neoadjuvant chemotherapy in locally advanced rectal cancer. J Gastroenterol Hepatol 34(1):140-146

27. Takayama Y, Nishie A, Togao O, et al. (2018) Amide proton transfer MR imaging of endometrioid endometrial adenocarcinoma: association with histologic grade. Radiology 286(3):909-917

28. Meng N, Fang T, Feng P, et al. (2021) Amide proton transfer-weighted imaging and multiple models diffusion-weighted imaging facilitates preoperative risk stratification of early-stage endometrial carcinoma. J Magn Reson Imaging 54:1200-1211

29. Li Y, Lin CY, Qi YF, et al. (2020) Non-invasive differentiation of endometrial adenocarcinoma from benign lesions in the uterus by utilization of amide proton transfer-weighted MRI. Mol Imaging Biol 23:446-455

30. Li Y, Lin CY, Qi YF, et al. (2021) Three-dimensional turbo-spin-echo amide proton transfer and intravoxel incoherent motion MR imaging for type I endometrial carcinoma: correlation with $\mathrm{Ki}-67$ proliferation status. Magn Reson Imaging 78:18-24

31. Togao O, Keupp J, Hiwatashi A, et al. (2017) Amide proton transfer imaging of brain tumors using a self-corrected 3D fast spin-echo dixon method: comparison with separate B correction. Magn Reson Med 77(6):2272-2279

32. He YL, Li Y, Lin CY, et al. (2019) Three-dimensional turbo-spin-echo amide proton transfer-weighted mri for cervical cancer: A preliminary study. J Magn Reson Imaging 50(4):1318-1325

33. Pecorelli S (2009) Revised FIGO staging for carcinoma of the vulva, cervix, and endometrium. Int J Gynaecol Obstet 105(2):103-104

34. Doghri R, Houcine $Y$, Boujelbène N, et al. (2019) Mismatch repair deficiency in endometrial cancer: immunohistochemistry staining and clinical implications. Appl Immunohistochem Mol Morphol 27(9):678-682

35. Kandoth C, McLellan MD, Vandin F, et al. (2013) Mutational landscape and significance across 12 major cancer types. Nature 502(7471):333-339 
36. Yamashita H, Nakayama K, Ishikawa M, et al. (2018) Microsatellite instability is a biomarker for immune checkpoint inhibitors in endometrial cancer. Oncotarget 9(5):5652-5664

37. Katz V, Lentz G, Lobo R, Gershenson D (2007) Diagnostic procedures. Imaging, endometrial sampling, endoscopy: indications and contraindications, complications, Comprehensive Gynecology, 5th edn. Mosby Elsevier, Philadelphia

38. Luomaranta A, Leminen A, Loukovaara M (2015) Magnetic resonance imaging in the assessment of high-risk features of endometrial carcinoma: a meta-analysis. Int J Gynecol Cancer 25(5):837-842

39. Fan Z, Li H, Hu R, Liu Y, Liu X, Gu L (2018) Fertility-preserving treatment in young women with Grade 1 presumed Stage IA endometrial adenocarcinoma: a meta-analysis. Int J Gynecol Cancer 28(2):385-393

40. Minamiguchi K, Takahama J, Uchiyama T, et al. (2018) Uterine endometrial carcinoma with DNA mismatch repair deficiency: magnetic resonance imaging findings and clinical features. Jpn J Radiol 36(7):429-436

41. Lu KH, Dinh M, Kohlmann W, et al. (2005) Gynecologic cancer as a "sentinel cancer" for women with hereditary nonpolyposis colorectal cancer syndrome. Obstet Gynecol 105(3):569-574

\section{Publisher's Note}

Springer Nature remains neutral with regard to jurisdictional claims in pub-

lished maps and institutional affiliations.

\section{Submit your manuscript to a SpringerOpen ${ }^{\circ}$ journal and benefit from:}

- Convenient online submission

- Rigorous peer review

- Open access: articles freely available online

- High visibility within the field

- Retaining the copyright to your article

Submit your next manuscript at $\boldsymbol{\nabla}$ springeropen.com 